Cahiers $d u$ MONDE RUSSE

\section{Cahiers du monde russe}

Russie - Empire russe - Union soviétique et États indépendants

$53 / 2-3 \mid 2012$

L'invention de la Sainte Russie

\title{
Le moine-peintre et le primitif
}

L'invention des « Primitifs » russes dans une perspective internationale Monk-painters and primitives: The invention of Russian "primitives" in international perspective

\section{François-René Martin}

\section{OpenEdition}

Journals

Édition électronique

URL : http://journals.openedition.org/monderusse/9395

DOI : $10.4000 /$ monderusse.9395

ISSN : $1777-5388$

Éditeur

Éditions de l'EHESS

Édition imprimée

Date de publication : 15 septembre 2012

Pagination : 467-477

ISSN : 1252-6576

Référence électronique

François-René Martin, «Le moine-peintre et le primitif », Cahiers du monde russe [En ligne], 53/2-3 | 2012, mis en ligne le 01 juillet 2015, Consulté le 26 avril 2019. URL : http://

journals.openedition.org/monderusse/9395; DOI : 10.4000/monderusse.9395

Ce document a été généré automatiquement le 26 avril 2019 


\title{
Le moine-peintre et le primitif
}

\author{
L'invention des « Primitifs » russes dans une perspective internationale \\ Monk-painters and primitives: The invention of Russian "primitives" in \\ international perspective
}

François-René Martin

1 En 1904 le peintre d'icônes Gurianov est invité par l'higoumène du monastère de la Trinité Saint-Serge à nettoyer les icônes qui y sont conservées. L'une d'entre elles est déjà célèbre, mais pour des raisons d'ordre historiographique: elle est la seule œuvre identifiée avec certitude, parmi quarante attribuées, à un moine-peintre du monastère Saint-Andronikos de Moscou, qui collabora avec un peintre byzantin, Théophane le Grec, dans les églises du Kremlin à Moscou en 1405. Ce moine-peintre, il s'agit d'Andrej Rublev, a peint peu avant sa mort survenue en 1430, à la demande de Nikon de Radonège, une icône de la Trinité à la gloire de saint Serge ${ }^{1}$. L'invention de cette œuvre ressemble de prime abord à d'autres découvertes, faisant passer une icône qui était déjà célèbre chez les spécialistes au rang de chef-d'œuvre de l'art russe, admiré par un public plus large. L'opération d'invention s'accomplit par toute une série d'interventions matérielles: l'enlèvement de l'oklad et le nettoyage de l'œuvre, qui permit de retrouver, sous plusieurs couches de repeints, la peinture originale, débarrassée d'une gaine dorée, et qui put apparaître ainsi dans son évidente beauté. L'icône était devenue une peinture ; le moine Rublev un " primitif ». Quant au public qui se pressa alors dans l'église de la Trinité, il ne venait plus tant vénérer une image religieuse mais une peinture de musée ${ }^{2}$.

2 Cette invention est-elle si différente de celle d'autres peintures de Primitifs, comme celles, longtemps oubliées ou négligées, des Primitifs français? Déjà, la concomitance de quelques-unes des découvertes les plus spectaculaires est éloquente. 1904 est en effet l'année où, au Louvre, dans le pavillon de Marsan, et à la Bibliothèque nationale, fut organisée l'exposition parisienne des Primitifs français ${ }^{3}$. Il s'agissait alors d'une exposition de combat, visant à répliquer aux Belges qui avaient organisé à Bruges, deux ans auparavant, la mémorable exposition des Primitifs flamands, et aux Italiens qui avaient célébré leurs propres Primitifs à Sienne. Le commissaire de l'exposition française, Henri Bouchot, cherchait avant tout à montrer qu'il existait une école spécifiquement 
française de peinture au $\mathrm{xv}^{\mathrm{e}}$ siècle, indépendante de l'école flamande non d'un strict point de vue stylistique mais tout au moins d'un point de vue social - c'est le terme qu'il utilisait - et économique. Si le fondement intellectuel qui justifiait cette exposition fut, pour l'essentiel, mis en pièce par la critique, la manifestation en tant que telle fut une véritable réussite, voyant des foules de curieux, d'amateurs, d'artistes se presser pour découvrir des œuvres qui étaient dans l'ensemble méconnues, parfois presque totalement inconnues en dehors du cercle des érudits: ainsi l'Annonciation d'Aix, une des œuvres phares de l'exposition, L'Homme au verre de vin, ou encore la Vierge d'Anvers de Jean Fouquet, qui suscita cependant des jugements négatifs de la part du comte Durieu ou de Huysmans. S'il est cependant une œuvre que l'exposition révéla, plus que tout autre, c'est bien la Pietà d'Avignon, une œuvre qui avait moins attiré les regards, à Villeneuve-lèsAvignon, que le Couronnement de la Vierge d'Enguerrand Quarton, clairement dissociée à cette époque de la Pietà. Cette dernière, en effet, était alors une œuvre anonyme qui, d'œuvre cultuelle, était passée rapidement au statut d'œuvre muséale, acquise par le Louvre l'année suivante, devenant même une des œuvres les plus célèbres de la peinture française ${ }^{4}$.

3 Une autre œuvre fut en quelque sorte inventée en 1904 : le Retable d'Issenheim, et en premier lieu les peintures de Grünewald. Le polyptyque, certes, n'était pas inconnu : il attirait les regards depuis le milieu du XIx ${ }^{e}$ siècle, mais il n'était pas considéré comme une œuvre de premier rang. Juste avant 1870, Alfred Woltmann l'avait hissé au rang de chefd'œuvre de l'art allemand, mais cela n'en faisait dans son esprit qu'un chef-d'œuvre parmi d'autres œuvres insignes ${ }^{5}$. En 1891, dans son roman Là-Bas, Huysmans avait attiré l'attention sur une peinture de l'artiste appartenant à un autre retable : la Crucifixion de Kassel. Mais il faut en réalité attendre 1904-1905, puis 1917 et le transfert à Munich à l'Alte Pinakothek, pour que le retable colmarien soit réputé chef-d'œuvre susceptible de représenter l'art allemand dans sa totalité. Ce changement de statut et de nature du Retable d'Issenheim n'a pas dépendu d'une exposition mais s'est en quelque sorte cristallisé dans un voyage et un texte. Entre la visite des expositions des Primitifs de Bruges et de Paris, Huysmans avait décidé de faire le voyage de Colmar, pour voir les Grünewald. Il avait rendu compte de cette visite et de la contemplation des panneaux du Retable d'Issenheim dans un texte testament, Trois Primitifs, publié en 1904, dans lequel il faisait du polyptyque un chef-d'œuvre absolu, un au-delà de la peinture ${ }^{6}$. Immense et très rapide fut l'impact de la description de Huysmans en Allemagne, où le crédit de la redécouverte, chez les expressionnistes, fut le plus souvent donné à Huysmans ${ }^{7}$. Quoi qu'il en soit du rôle joué par Huysmans, il est assez intéressant de voir que, là encore, la redécouverte d'un chef-d'œuvre que l'on n'avait pas de peine, à ce moment, à classer parmi les «Primitifs », s'était en quelque sorte cristallisée en 1904.

\section{D'un primitif à l'autre : Henri Matisse et les icônes russes}

4 Ces trois « redécouvertes » doivent être bien entendu replacées dans un mouvement plus général, qui se déploie en réalité sur près d'un siècle. Elles relèvent du mouvement que Francis Haskell appelait la "seconde découverte des maîtres anciens » autour de $1900^{8}$. À la première vague de «redécouverte » des maîtres anciens, et notamment des Primitifs, autour de 1800, qui avait ouvert une longue période de réévaluation des périodes les plus anciennes de la peinture et de redistribution des valeurs, succède une seconde vague, 
marquée par de grandes expositions, mais aussi par l'invention de chefs-d'œuvre restés en quelque sorte en réserve de la critique. Des chefs-d'œuvre difficiles en outre, que le goût primitiviste dominant ne pouvait, jusqu'à la fin du XIX ${ }^{\mathrm{e}}$ siècle, aisément assimiler. C'est ainsi que les tableaux d'Enguerrand Quarton ou du maître de Moulins, longtemps vus comme des développements faibles ou périphériques de l'art flamand, furent à ce moment considérés comme des aboutissements de l'art français; les peintures de Grünewald jusque-là perçues comme "gothiques", c'est-à-dire attardées, vinrent concurrencer et même se substituer à celles de Dürer dans la définition de la germanité. Quant aux icônes russes, elles deviennent dans l'œil des historiens - par exemple Nikolaj Lihačev dans sa Manière de peindre d'Andrej Rublev (1906) - des peintures justiciables des méthodes du connoisseurship, vite convertibles dans la catégorie des primitifs. Mais si ces "redécouvertes» dépendent chacune de circonstances singulières et de contextes nationaux particuliers, elles s'inscrivent dans une véritable dynamique internationale, où chaque nation vient faire valoir ses droits, où chaque collectionneur, connaisseur, savant, artiste à la recherche des sources les plus pures de la peinture est aspiré dans un mouvement incessant de compétition dans le primitivisme et de redistribution de ses valeurs.

5 L'exemple de Matisse l'illustre d'une façon presque idéale. Avec Derain, Matisse avait été fasciné par les maîtres anciens montrés à Paris en 1904. Il était même allé enterrer un de ses jeunes amis, Linaret, mort d'un infarctus dans les salles du pavillon de Marsan, devant L'homme au verre de vin'. Lorsqu'il vient rendre visite à Hugo von Tschudi en 1910 à Munich, le même Matisse a un œil sur Grünewald. Alors que l'on débat chez Tschudi des mérites de Gauguin et de Van Gogh, Matisse pointe la faiblesse en composition de ce dernier en comparant avec La Dérision qui se trouvait dans la salle voisine ${ }^{10}$. L'œuvre avait été découverte et attribuée en 1909, restaurée en 1910 pour venir enrichir la collection de l'Alte Pinakothek, placée à côté d'un autre chef-d'œuvre de Grünewald, le Saint Erasme et saint Maurice. L'année suivante, Matisse découvre, alors qu'il visite Moscou à l'invitation de Sergej Ščukin, les chapelles des vieux croyants et l'art des icônes. Il déclare à cette occasion :

Je connais l'art religieux de plusieurs pays, mais nulle part je n'ai vu une telle révélation du sentiment mystique, parfois même de l'effroi religieux. [...]. Les icônes, quant à elles, sont un échantillon des plus intéressants de la peinture primitive [...] Je n'ai vu nulle part une telle pureté des couleurs, une telle spontanéité de la représentation. C'est le meilleur patrimoine de Moscou. Il faut venir ici s'instruire, car c'est chez les primitifs qu'il convient de chercher l'inspiration. ${ }^{11}$

En six ans, Matisse avait en quelque sorte traversé l'espace à la fois raréfié et commun des primitifs les plus difficiles, soudainement révélés au public, et cela dans trois scènes distinctes.

7 La découverte faite par Matisse s'inscrivait alors dans un mouvement général de réintégration esthétique, six ans après les enthousiasmes des nouveaux pèlerins du vieil art russe. Pour peu que nous la mettions dans la perspective de ses engouements pour les primitifs des différentes scènes, elle prend une signification particulière : comme si les icônes russes pouvaient figurer l'art le plus achevé de la peinture primitive et en même temps l'art qui n'aurait été révélé que tardivement. Le dernier moment de ces revivals des années 1900-1910 et en même temps le dernier mot d'un primitivisme libératoire, clairement byzantin dans le cas de Matisse : 
J'ai compris la peinture byzantine devant les icônes à Moscou. On se livre d'autant mieux qu'on voit ses efforts confirmés par une tradition, si ancienne fût-elle. Elle vous aide à sauter le fossé. ${ }^{12}$

\section{La concurrence dans les musées}

Ces enchères primitivistes, dans l'œil de Matisse, ne sont que la traduction d'une compétition internationale dans la production et la promotion de primitifs nationaux. Une compétition qui implique de la manière la plus forte les institutions, qu'il s'agisse des musées ou des expositions. Les mutations du goût, au XIX ${ }^{\mathrm{e}}$ siècle, qui hissent les primitifs au sommet dans la hiérarchie artistique n'ont pas été simplement portées par les collectionneurs et les artistes, mais aussi par les musées et les historiens de l'art, dans une ambiance à la fois universaliste et nationaliste. Chaque nation aspire à avoir des collections qui reflètent sa vocation universaliste. S'il est un musée qui a constitué la matrice de ce modèle, c'est bien le Louvre tel qu'il fut constitué à la Révolution et sous l'Empire. Ce modèle va se transformer au long du XIX siècle, mais persistera, reprenant une certaine vigueur lorsque les sociétés impériales, à la fin du xix ${ }^{\mathrm{e}}$ siècle, chercheront à faire du musée une institution centrale de leurs capitales ${ }^{13}$. Le British Museum à Londres ou le Kunsthistorisches Museum de Vienne, le neues Museum de Berlin sont trois autres exemples de ce modèle. Face à ce modèle universaliste est le modèle national, qui met l'accent sur l'art autochtone, l'art créé dans le ressort national. C'est le Louvre, tel qu'il est redéfini après 1815, ou l'Alte Pinakothek de Munich après l'acquisition des primitifs de la collection Boisserée en 1827. Ces deux modèles peuvent s'articuler, se succéder dans un même musée, coexister dans une même nation. Ce qui rend toutefois possible leur coexistence est la nécessité qui se fait de plus en plus forte, dans la seconde moitié du XIX ${ }^{e}$ siècle, pour une nation ou un empire, de posséder de grands musées, avec des œuvres qui expriment la puissance extérieure mais aussi avec des œuvres qui attestent d'une tradition picturale ancienne et pure ${ }^{14}$. Tel est le fondement idéologique de la constitution ou de l'enrichissement des grandes collections de primitifs, particulièrement entre 1870 et 1930. Plus peut-être que les grandes bibliothèques ou même les grands opéras qui forment autant de lieux phares dans les capitales qui se développent à ce moment, le musée est un attribut de la puissance étatique, du rayonnement des capitales mais aussi de la cohérence nationale. Les nations rivalisent dans l'accumulation des collections et le gigantisme des bâtiments, symboles de puissance, mais restent soumises à une opération infiniment délicate qui est de circonscrire et protéger de toute influence extérieure une sorte de « réserve » artistique ou patrimoniale, comme il existe dans les États une réserve monétaire ou un stock d'or. Dans cette course à l'accumulation et à la singularisation, les cas que forment la Russie et les États-Unis sont des plus intéressants. La naissance du Musée russe de Saint-Pétersbourg illustre le phénomène d'alignement dans la compétition; la construction de grands musées à la fin du xix ${ }^{e}$ siècle dans de grandes villes comme Philadelphie et la constitution pendant la première moitié du XIX ${ }^{\mathrm{e}}$ siècle de collections de primitifs de premier plan illustrent enfin le conformisme des sociétés impériales, qui fait qu'une nation peut importer des primitifs sur son sol, à des milliers de kilomètres de leur foyer, créer comme Isabella Stewart Gardner à Boston un musée dans un palazzo néo-florentin, pour manifester sa vocation universaliste, sinon un style de vie international ${ }^{15}$. En Russie et en Amérique, comme autrefois en Allemagne, en Italie ou en Angleterre, les Primitifs sont les trophées les plus marquants des musées. À la faveur de la rehiérarchisation des valeurs artistiques, ce vaste mouvement de fond - la préférence 
pour le primitif comme le formulait E.H. Gombrich -, aboutit à ce que les peintures jadis décriées comme gothiques ou byzantines deviennent l'expression la plus pure de l'âme des nations. C'est ainsi que la Russie peut opposer aux autres nations ses propres primitifs, enfin visibles, débarrassés des repeints, des oklads, des regards dévots, aptes à concourir dans une compétition destinée à remplir les salles de musées de peintures des « maîtres anciens d'État », comme se plaît à l'écrire Thomas Bernhard ${ }^{16}$.

\section{Conflits historiographiques et sentiment d'incomplétude nationale}

9 Cette compétition muséale au long du XIX siècle où la place des Primitifs est de plus en plus sensible - d'autant plus sensible qu'elle ouvre la collection en même temps qu'elle fonde un récit national -, se double inévitablement de luttes idéologiques intenses, assumées par les historiens de l'art, qui sont les plus qualifiés pour prendre en charge cette matière universelle et locale forcément problématique, mais qui apparaît comme étant de plus en plus importante dans la définition de la nation. C'est en Allemagne que cette opération d'indexation de l'histoire nationale sur l'histoire de l'art a été faite de la manière la plus sophistiquée, dès les années 1820-1830, au sein de l'École de Berlin, avec des historiens de l'art tels que Franz Kugler et Gustav F. Waagen qui conçoivent, l'un l'histoire universaliste de l'art, l'autre un musée national, avec pour idée que l'art, plus que la politique, est l'épine dorsale de l'histoire ${ }^{17}$. Hans-Robert Jauss a très bien caractérisé ce renversement essentiel dans l'historiographie, qui fait que l'art plus que la politique posséderait une haute qualité de consistance ou d'inhérence nationale ${ }^{18}$; ce renversement va fonder tous les conflits, dans l'histoire de l'art, qui vont diviser les spécialistes, notamment sur les Primitifs, pour qui chaque discussion érudite sera susceptible d'avoir simultanément une signification savante et politique et de correspondre ainsi à un enjeu disproportionné.

Il est difficile de résumer en quelques phrases tous ces conflits qui, dans la seconde moitié $\mathrm{du}$ xIX ${ }^{\mathrm{e}}$ siècle, touchent à l'histoire de l'art et notamment aux Primitifs. L'on peut néanmoins tenter d'énumérer et de caractériser les principaux :

- les conflits sur la priorité nationale dans la formation d'un style. Le plus aigu est sans doute celui qui touche au gothique que se disputent la France et l'Allemagne, dès les années 1840 ;

- les conflits sur la primauté dans l'accomplissement d'un style. Ainsi les historiens de l'art allemands qui veulent bien concéder que le gothique soit né en France ajoutent vite qu'il a trouvé son lieu d'épanouissement, de maturité, en Allemagne ;

- les conflits sur la continuité temporelle d'une tradition nationale. C'est ce qui fait que les Français se battent, vers 1900 , pour faire admettre qu'il existe une généalogie ininterrompue qui va de Fouquet à Poussin, David et Ingres ;

- les conflits qui touchent à la continuité stylistique territoriale ou encore, ce qui n'est pas tout à fait la même chose, la correspondance entre le territoire stylistique et le territoire politique. Le cas russe peut être cité: est-il possible de définir l'art d'un ensemble géographique aussi vaste que la Russie en se fondant sur le foyer limité qu'est Novgorod? ;

- les conflits qui touchent à la domination stylistique d'une nation sur une autre ou, ce qui est à peu près la même chose, à ce qu'un Louis Réau appellera dans les années 1920 l'expansion artistique. Là encore la Russie offre l'exemple d'une nation que les historiens d'art ont souvent décrit comme très passive, n'ayant jamais été qu'une terre d'expansion de styles puissants constitués ailleurs. Ainsi Louis Réau peut-il écrire dans son Art russe que ce dernier 
se montre inférieur par rapport aux arts des grands peuples de l'Orient et de l'Occident dès lors qu'il est « hybride », mi-européen, mi-asiatique, devant plus qu'aucun autre aux apports étrangers : «C'est un art sans rayonnement qui n'a jamais eu aucune expansion et n'a jamais essaimé au dehors. C'est un art retardataire qui, à partir du XIII ${ }^{\mathrm{e}}$ siècle, s'est toujours laissé distancer. Enfin, c'est un art qui, comparé à ceux d'Occident, nous semble discontinu et incomplet $[. . .]^{19}{ }^{\prime}$;

- Les conflits où est en jeu l'existence d'un cheminement stylistique spécifique à une nation. La thèse développée par Kurt Gerstenberg en 1913 sur le Sondergotik, le cheminement spécifiquement allemand de l'art gothique, indépendamment du gothique français et surtout de la Renaissance, est un modèle idéologique susceptible d'être projeté sur d'autres nations ${ }^{20}$ . De période, le gothique devient un caractère stylistique national persistant ou même permanent. Il en est de même, dans le cas russe, de l'art byzantin qui est tantôt compris comme une période de soumission à un idiome étranger précédant l'avènement de l'art spécifiquement russe, tantôt comme un substrat qui définirait en profondeur l'art novgodorien dans ce qu'il a de plus original.

11 Tous ces conflits, ces polémiques qui jalonnent l'historiographie dans la seconde moitié $\mathrm{du} \mathrm{XIX}^{\mathrm{e}}$ siècle et du début $\mathrm{du} \mathrm{Xx}^{\mathrm{e}}$ siècle, montrent en réalité que, dans la construction des imaginaires nationaux, l'utilisation de la matière artistique est délicate et source de perturbations, au moins dans le domaine historiographique. Le revers de la puissance muséale et de la clarification très spectaculaire qu'elle opère dans la différenciation des écoles et dans la redécouverte des Primitifs qu'elle vient célébrer, est la fragilité presque constitutive des montages historiographiques qui les complètent et tentent de les justifier intellectuellement. Cette fragilité s'observe de la manière la plus vive dans le cas des Primitifs français. En 1904, un Henri Bouchot, le commissaire de l'exposition de 1904, s'épuisera à démontrer que les Primitifs français étaient sinon stylistiquement, tout au moins économiquement indépendants des Primitifs flamands au $\mathrm{xv}^{\mathrm{e}}$ siècle et que l'École de Fontainebleau, avec ses grands émigrés italiens, ne définissait pas l'art français du XVI siècle. Autour de 1900, la communauté des historiens de l'art allemands se déchire autour du cas de Dürer, soupçonné d'avoir contaminé l'art allemand en important des motifs italiens lors de ses deux séjours présumés ${ }^{21}$. Ces deux exemples montrent combien, en Europe, particulièrement dans de grandes nations comme la France ou l'Allemagne, est présent, dans l'historiographie des Primitifs, un sentiment d'incomplétude nationale au sujet de leurs primitifs. Le cas russe manifeste lui aussi un véritable complexe d'incomplétude, mais d'un autre type. Il s'y ajoute la conscience d'un retard historiographique.

\section{Le travail de rattrapage historiographique : icônes ou Primitifs russes?}

12 Avant la redécouverte des icônes et des Primitifs russes, qui se produit dans les années 1904-1920, les historiens de l'art considéraient essentiellement leurs icônes sous leur aspect cultuel ou pour leur valeur historique. Le Manuel illustré du peintre d'icônes de Nikodim Kondakov, élève de Fedor Buslaev, publié à Saint-Pétersbourg en 1905, illustre cette vision encore marquée par la tradition de l'érudition sacrée, héritée de Didron. Mais cependant y apparaît l'idée que l'art des icônes, au $\mathrm{xv}^{\mathrm{e}}$ siècle, dépend étroitement de l'art italien, via l'école italo-crétoise ${ }^{22}$. Au même moment, quelques collectionneurs et quelques critiques considèrent sous un autre jour les icônes, appréciées pour leur haute 
valeur artistique, qui les rend désormais comparables avec les peintures des primitifs d'Europe occidentale sans les en faire dépendre. Enfin, et c'est un aspect essentiel de cette conversion, elles sont assimilées par les artistes et les collectionneurs avant-gardistes. Chez Morozov et Ščukin, les chefs-d'œuvre de Cézanne ou de Gauguin voisinent avec des icônes. Et bientôt de jeunes artistes, dès 1910, intègrent cette esthétique dans leur répertoire comme Mihail Larionov et Natalja Gončarova. Comme ce fut le cas pour les autres scènes, l'avant-garde russe trouva ses propres " primitifs ", ce qui permettait à la fois d'inventer une tradition nationale et de rejoindre le mainstream primitiviste international.

Dans ce travail historiographique de rattrapage, les questions liées à la parenté avec les primitifs occidentaux et à l'accomplissement d'une école spécifiquement nationale sont au cœur des préoccupations des collectionneurs et des érudits. Ainsi Ostrouhov peut-il faire voisiner, dans sa collection, une des plus marquantes de son temps, l'icône russe avec des Primitifs italiens. Cette réintégration des icônes chez des collectionneurs excentriques mais très influents, qui agissent en prescripteurs du goût, précède et autorise le mouvement critique qui va faire des peintres d'icônes les cousins plus ou moins éloignés des Primitifs italiens. Grand admirateur d'Ostrouhov, Pavel Muratov est sans nul doute l'historien de l'art chez qui toutes ces questions sont posées avec le plus d'acuité. Lecteur de Walter Pater et de Bernard Berenson, amoureux de Florence où il séjourne entre 1908 et 1911, Muratov insiste dès 1912 sur la nécessité de posséder un musée national russe, où seraient montrées les icônes :

Nous n'avons pas de musée national où soit réunie de façon honorable notre incomparable peinture d'icônes, où soit exposée la beauté ancienne de l'église russe, de la maison marchande russe, de la gentilhommière russe. ${ }^{23}$

Restait alors à faire accéder les icônes à un tout autre niveau de grandeur historiographique, ce que Muratov s'emploiera à faire en premier lieu dans le volume sur la peinture ancienne que lui confie Igor' Grabar, éditeur d'une Histoire de l'art russe. Dans Les Icônes russes, publié en 1927, Muratov expose son schéma de développement de l'ancienne peinture russe, dont la source se trouve dans l'art byzantin. Un art byzantin qui n'aurait pas été un art enfermé dans des formules rigides, mais connaissant des moments de réveil, comme la "Renaissance byzantine» aux $\mathrm{x}^{\mathrm{e}}$-XII ${ }^{\mathrm{e}}$ siècles, qui coïnciderait avec la première époque de la civilisation russe. Essentiellement byzantine et grecque dans cette première période, la peinture russe n'est plus une province de Byzance au XIV siècle. S'appuyant sur les travaux de Millet et Diehl, Muratov en vient alors à voir dans la dernière Renaissance byzantine, au XIV siècle, un mouvement parallèle de celui de l'art toscan, qui suivit ses voies particulières et vint nourrir l'art russe $^{24}$. Ce n'est qu'au Xv ${ }^{\mathrm{e}}$ siècle et non un siècle auparavant comme le pensait Kondakov qu'existe une école italo-crétoise influente en Orient, dont dépendent des icônes qui s'opposeraient très distinctement aux icônes russes qui leur sont contemporaines.

\section{Byzance face à Sienne}

Cette filiation gréco-byzantine, loin de toute influence italienne, est ce qui permit à l'art russe de se révéler, selon Muratov, dans toute sa véritable grandeur. "Grâce à son isolement de l'Europe, l'ancienne Russie garda le secret d'une peinture qui se base sur des conceptions et des procédés qu'on ne rencontre guère dans l'art de la Renaissance européenne $e^{25}$.» C'est ce qui lui a permis de conserver, plus directement qu'ailleurs, «la 
tradition encore plus lointaine et plus belle [de] la peinture de l'antiquité classique $»^{26}$. Mais pour autant, cet effort de construction d'une généalogie spécifique, préservée de toute influence italienne, n'empêche pas Muratov de recourir à des confrontations visuelles. Comme Ostrouhov le faisait dans sa collection, Muratov multiplie dans l'espace du livre, particulièrement les Trente-cinq Primitifs russes, les rapprochements purement formels permettant de faire résonner des noms parmi les plus consacrés de l'art siennois avec des œuvres russes ${ }^{27}$. Une icône de l'école de Novgorod, avec son paysage sommaire et ses rochers en escalier, est rapprochée d'une peinture de Duccio. Autre confrontation : la Vierge du Don, alors attribuée à Théophane le Grec, et un des fleurons de la collection Stroganov, aujourd'hui au Metropolitan Museum of Art à New York, la Vierge à l'Enfant de Duccio. Pour Muratov, qui se fondait sur les travaux de Kallab, il s'agissait donc d'un héritage antique, que l'on trouvait aussi bien dans l'art italien que dans l'art des icônes, où il survivait avec une plus grande pureté. Mais au-delà des constructions généalogiques, tous ces rapprochements, chez un historien de l'art qui devait écrire une monographie sur Fra Angelico ${ }^{28}$, montraient bien que la porte d'accès, en matière de goût, à ces "Primitifs russes ", avait bien été celle de Sienne et de Florence.

Restait à voir si les historiens de l'art des autres pays, et notamment les spécialistes des Primitifs, allaient valider cette réintégration originale et complexe. Préfacier des Trentecinq Primitifs, Henri Focillon restait somme toute assez évasif sur la question du développement séparé de l'art occidental et de l'art russe, se contentant d'écrire que l'Orient et l'Occident chrétiens ne constituaient pas «deux mondes symétriques et opposés, de chaque côté d'un arbre de vie ou d'un pyrée, mais qu'ils étaient unis par des échanges et que certains caractères communs de leur art pouvaient s'expliquer soit par le jeu des relations réciproques, soit par la communauté des origine ${ }^{29}$ ». Dans son Art russe publié à Paris en 1921 et 1922, Louis Réau - qui ne manque pas de rappeler l'importance de Didron dans la redécouverte des icônes russes - valide pour l'essentiel la thèse byzantinisante de Muratov $^{30}$. Les italianismes de l'art novgorodien ne tiennent qu'à cette source commune. Byzance et non Sienne : en arbitrant dans cette querelle, Réau donnait plus de poids aux thèses qui déplaçaient les origines de l'art occidental vers l'Orient. Il ne faisait que souligner là la manière, très efficace, dont les érudits russes étaient parvenus à imposer leur propre idée des Primitifs : un art aussi abouti que le grand art siennois, mais qui devait tout à Byzance et était resté ainsi original et spécifique.

\section{NOTES}

1. Voir Pierre Gonneau, À l'aube de la Russie moscovite: Serge de Radonège et Andreï Roublev, Légendes et images (XIV'-XVII siècles), P. : Bibliothèque russe de l'Institut d'études slaves, CXIV, Institut d'études slaves, 2007.

2. Sur cette invention et plus généralement celle des icônes russes, une introduction limpide se trouve chez Olga Medvedkova, Les icônes en Russie, P. : Gallimard, 2010.

3. Exposition des Primitifs français au Palais du Louvre (pavillon de Marsan) et à la Bibliothèque nationale, cat. exp., P.: Palais du Louvre et Bibliothèque nationale, 1904 ; Henri Bouchot, 
Les Primitifs français 1292-1500. Complément documentaire au catalogue de l'exposition, P., 1904. Sur cette exposition et les enjeux idéologiques qui l'entouraient, nous nous permettons de renvoyer à: Dominique Thiébaud, Philippe Lorentz, François-René Martin, Primitifs français : Découvertes et redécouvertes, cat. exp., P. : Musée du Louvre, 2004 ; F.-R. Martin, «L'administration du génie national. L'exposition des primitifs français de 1904 », in Enrico Castelnuovo, Alessio Monciatti, éds., Medioevo/Medioevi: Un secolo di esposizioni d'arte medievale, Pise : Edizioni della Normale, 2008, p. 93-108.

4. Voir Charles Sterling, Enguerrand Quarton : Le peintre de la Pieta d'Avignon, P. : Éditions de la Réunion des musées nationaux, 1983, p. 81 sq.

5. Alfred Woltmann, "Ein Hauptwerk deutscher Kunst auf französischem Boden », Zeitschrift für bildende Kunst, I, 1866, p. 257-262, 283-287 ; A. Woltmann, «Streifzüge im Elsaß V. Der deutsche Corregio ", Zeitschrift für bildende Kunst, VIII, 1873, p. 321-331. Sur l'historiographie de Grünewald et ses « redécouvertes » : F.-R. Martin, « Grünewald et ses critiques ( $\mathrm{xvI}^{\mathrm{e}}-\mathrm{XXI}^{\mathrm{e}}$ siècles)», thèse d'histoire de l'art, université Marc-Bloch de Strasbourg, 3 vol., 2008.

6. Joris Karl Huysmans, "Les Grünewald du musée de Colmar », Le mois littéraire et pittoresque, 63, mars 1904, p. 282-300 ; J.K. Huysmans, Trois Primitifs, P. - Messein, 1905.

7. Voir J.K. Huysmans, Geheimnisse der Gotik: Drei Kirchen und drei Primitive, Munich Berlin : Georg Müller, 1918 ; Ingrid Schulze, Die Erschütterung der Moderne: Grünewald im 20. Jahrhundert, Leipzig : E.A. Seemann, 1991, p. 8-15.

8. Francis Haskell, L'amateur d'art, P. : Le Livre de poche, 1997, p. 40-89.

9. Roger Benjamin, Matisse's "Notes of a Painter": Criticism, Theory, and Context, 1891-1908, Ann Arbor : UMI Research Press, 1987, p. 31-34.

10. La discussion est rapportée par Alfred H. Barr, Matisse: his Art and his Public, New York: The Museum of Modern Art, 1951, p. 109.

11. Henri Matisse, interview à Russkie Vedomosti, 27 octobre 1911, cité par O. Medvedkova, Les icônes en Russie, p. 18-19.

12. «Témoignage » recueilli par Gaston Diehl dans Peintres d'aujourd'hui, collection Comoedia-Charpentier, juin 1943, repris dans Dominique Fourcade, éd., Henri Matisse, Écrits et propos sur l'art, P. : Hermann, 1972, p. 196. Sur le byzantinisme de Matisse, voir Rémi Labrusse, Matisse : La condition de l'image, P. : Gallimard, 1999, p. 81-83, 94-115.

13. Voir notamment les différents essais réunis par Christophe Charles, Le Temps des capitales culturelles (XVIII $-\mathrm{XX}^{e}$ siècles), Seyssel : Champ Vallon, 2009.

14. Ces tensions sont au centre des essais réunis par Gwendolyn Wright, éd., The Formation of National Collections of Art and Archaeology, Washington: National Gallery of Art, Hannover - Londres : The University Press of New England, 1996.

15. Question qu'étudie notamment Andrée Hayum, «L'idée de la Renaissance à l'aube du musée européen : les écoles primitives ", "The Migration of the Renaissance Primitives and the Early Public Museum in the Anglo-American World», communications dans le séminaire d'Antoine Compagnon, Paris, Collège de France, 18 et 25 mars 2010.

16. Thomas Bernhard, Maîtres anciens: Comédie, P.: Gallimard, 1988, p. 45-46. Voir à ce sujet notre essai : F.-R. Martin, " "Maîtres anciens d'État”. Quelques remarques sur l'histoire des expositions de maîtres anciens ", in Dominique Viéville, éd., Histoire de l'art et musées, P. : École du Louvre, 2005, p. 113-125. 
17. Ce qu'a bien montré Peter Paret, Art as History: Episodes in the Culture and Politics of Nineteenth-Century Germany, Princeton, Princeton University Press, 1988. Voir par ailleurs Gabriele Bickendorff, Der Beginn der Kunstgeschichtsschreibung unter dem Paradigma "Geschichte". Gustav Friedrich Waagens Frühschrift "Ueber Hubert und Johann Van Eyck", Worms : Wernersche Verlagsgesellschaft, 1985.

18. Hans Robert Jauss, Pour une esthétique de la réception, P. : Gallimard, 1978, p. 81-122.

19. Louis Réau, L’Art russe, rééd. 3 vol., P.: Marabout université, 1968, vol. 1, p. 32 (1 ${ }^{\text {re }}$ éd., P. : Henri Laurens, 1921-1922).

20. Voir notamment Hans Belting, The Germans and their Art: A Troublesome Relation, New Haven - Londres : Yale University Press, 1998, p. 49-60.

21. Voir Jan Bialostocki, Dürer and his Critics, 1500-1971, Baden Baden: Valentin Koerner 1986, p. 309 sq.

22. Idée prolongée dans: Nikodim Pavlovich Kondakov, The Russian Icon, Oxford: Clarendon Press, 1927. Et on lira: Ivan Foletti, Da Bisanzio alla Santa Russia: Nikodim Kondakov (1844-1925) e la nascita della storia dell'arte in Russia, Roma : Viella, 2011, qui discute par ailleurs les grandes lignes de notre essai, tel qu'il avait été présenté à l'occasion du colloque du musée du Louvre.

23. P. Muratov, «Muzej izjaščnih iskusstv v Moskve [Le musée des Beaux-Arts à Moscou] ", Apollon, SPb., n 9, 1912, p. 49, cité par Danièle Beaune-Gray, "L'itinéraire intellectuel de Pavel Muratov (1881-1945) », colloque Les premières rencontres de l'Institut européen Est-Ouest, Lyon, ENS LSH, 2-4 décembre 2004, p. 439-458, p. 444 (http://russieeurope.ens-lsh.fr/article.php3?id_article=46).

24. P.P. Muratov, Les Icônes russes, P. : J. Schiffrin, Éditions de la Pléiade, 1927, p. 90-98.

25. Ibid., p. 101.

26. Ibid., p. 102.

27. P. Muratov, Trente-cinq Primitifs russes. Collection Jacques Zolotnitzky, P.: À la Vieille Russie, 1931.

28. P. Muratoff, Fra Angelico, P. : Crès, 1929.

29. Henri Focillon, « Préface » à Muratov, Trente-cinq Primitifs russes,

30. Réau, L'art russe, 1968, vol. 1, p. 211-218.

\section{RÉSUMÉS}

La "redécouverte" des vieux peintres russes et des icônes est contemporaine de ce que l'on appelle la seconde redécouverte des primitifs en Europe, autour de 1900. Qu'il s'agisse des peintres français, allemands ou même italiens $d u x^{e}$ siècle ou du début du $x^{2} I^{e}$ siècle, ils devinrent, grâce à des expositions d'envergure à visée nationaliste, comme l'exposition des Primitifs français de 1904, les principaux garants de l'existence d'écoles spécifiquement nationales de peinture. Dans ce vaste mouvement de publicisation, qui suivit une phase de redécouverte strictement érudite, les collectionneurs et les artistes de l'avant-garde ont pu jouer 
un rôle essentiel. Or ce mouvement, qui a été souvent étudié nation par nation, est en réalité aussi international que concurrentiel. La Russie relève de ce mouvement, mais avec des spécificités qui tiennent tant aux caractéristiques des œuvres elles-mêmes qu'aux constructions intellectuelles des principaux historiens de l'art russe, celles, particulièrement, de Pavel Muratov.

Monk-painters and primitives: The invention of Russian "primitives" in international perspective The "rediscovery" of early Russian painters and icons was contemporaneous with the second rediscovery of primitive art that took place in Europe around 1900. Large-scale exhibitions serving nationalist purposes turned fifteenth- or early sixteenth-century French, German or even Italian painters into tokens of the existence of truly national schools of painting. A good example of this was the 1904 exhibition of the French Primitives. Art collectors and avant-garde artists played an essential role in this vast publicizing campaign which followed the rediscovery of these works by scholars. However, even though this movement has often been studied nation by nation, it was as international as it was competitive. Russia pertained to it while displaying specific features due as much to the characteristics of the works themselves as to the intellectual constructs of major Russian art historians, particularly Pavel Muratov.

\section{AUTEUR}

\section{FRANÇOIS-RENÉ MARTIN}

École nationale supérieure des Beaux-Arts, Paris 